\title{
Enhanced marking process (EMP) for constructing dominating set in mobile ad-hoc networks with unidirectional links
}

\begin{abstract}
Broadcasting or flooding is one of the principal functions in wireless ad-hoc networks. In broadcasting, a mobile node sends the same message to all nodes in the network in one-to-all model. Broadcasting based on set of dominating nodes is remunerative approach, where the broadcasting activity is constrained to only the nodes in the dominating set. A set is dominating if all nodes in the network are either in the set or neighbors of nodes in the set. In this study, the notion of constructing connected-dominating-set is extended to ad-hoc networks with unidirectional links. An enhanced distributed algorithm is presented that is based on the marking process which is has been introduced in earlier work. Our enhanced algorithm features a good locality properties since it need only 2-hop neighborhood information within each node. The algorithm checks for the mutual existence of nodes in the neighbor table of their neighbors to guarantee the symmetric connectivity between neighboring nodes. The proposed algorithm is integrated with AODV routing protocol to generate a connected dominating set that will be responsible on flooding activity. The efficiency of our approach is investigated and verified through simulation whereas the computational complexity is determined and compared with that of original marking process. All the simulations run are carried out with QualNet Simulator version 5.02.
\end{abstract}

Keyword: Ad hoc networks; Connected-dominating-sets; Flooding; Marking process; Unidirectional links; Virtual backbone 